
18

\title{
Identification of key genes and pathways associated with Crohn's disease by bioinformatics analysis
}

Zheng Wang ${ }^{1}$,Jie Zhu $^{1}$,Lixian $\mathrm{Ma}^{1}$

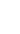

(1)

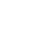

.

0

11

5 'Department of Infectious Diseases, Qilu Hospital, Shandong University, Shandong, China;

Corresponding author: Lixian Ma, E-mail: mlx_sdu@163.com 19 


\section{Abstract}

Crohn's disease is a type of inflammatory bowel disease posing a significant threat to human health all over the world. Genome-wide gene expression profiles of mucosal colonic biopsies have provided some insight into the molecular mechanisms of Crohn's disease. However, the exact pathogenesis is unclear. This study aimed to identify key genes and significant signaling pathways associated with Crohn's disease by bioinformatics analysis. To identify key genes, an integrated analysis of gene expression signature was conducted using a robust rank aggregation approach. A total of 179 Crohn's disease patients and 94 healthy controls from nine public microarray datasets were included. MMP1 and CLDN8 were two key genes screened from the differentially expressed genes. Connectivity Map predicted several small molecules as possible adjuvant drugs to treat $C D$. Besides, we used weighted gene co-expression network analysis to explore the co-expression modules associated with Crohn's disease pathogenesis. Seven main functional modules were identified, of which black module showed the highest correlation with Crohn's disease. The genes in black module mainly enriched in Interferon Signaling and defense response to virus. Blue module was another important module and enriched in several signaling pathways, including extracellular matrix organization, inflammatory response and blood vessel development. There were also several other 
42 meaningful functional modules which enriched in many biological

43 processes. The present study identified a number of key genes and 44 pathways correlated with Crohn's disease and potential drugs to combat

45 it, which might offer insights into Crohn's disease pathogenesis and 46 provide a clue to potential treatments.

47

48

49

50

51

52

53

54

55

56

57

58

59

60

61

62

63 


\section{Introduction}

Crohn's disease (CD) is a chronic nonspecific inflammatory bowel disease (IBD), which may affect any region of the gastrointestinal tract intermittently with the terminal ileum and colon being the most common [1]. The incidence and prevalence of $C D$ were highest in developed countries, with the highest annual incidence in North America (20.2 per 100,000$)$ and Europe $(12.7$ per 100,000$)$. Similarly, the highest worldwide prevalence of CD was found in Europe $(322$ per 100,000) and North America (319 per 100,000) [2]. Furthermore, the incidence of CD in the developing countries continues to increase. Regarding sexual distribution, there was no sex preponderance in adult CD [1]. About $12 \%$ of $C D$ patients have a family history of IBD, with an even higher proportion of family cases in younger individuals [3]. Although the exact etiology of $C D$ remains uncertain to date, there is evidence that it involves a complex interaction between the individual's genetic susceptibility, external environment, intestinal microbial flora and the immune responses $[4,5]$. Previous genome-wide association studies have reported numerous loci associated with CD, which included NOD2, ATG16L1 and IRGM. NOD2 was identified as the first susceptibility gene for CD [6]. NOD2, a cytosolic pattern recognition receptor for the bacterial proteoglycan fragment muramyl dipeptide, plays a significant role in maintaining immunological homeostasis in the intestine. NOD2 
mutations are genetically correlated with an increased risk for the development of $\mathrm{CD}[5,7]$. Moreover, genetic analyses have identified autophagy genes ATG16L1 and IRGM genes as susceptibility genes for CD [8]. Together, these common susceptibility genes account for $13.1 \%$ of variance in disease liability in CD [9].

A large amount of gene expression datasets are currently freely available from the Gene Expression Omnibus (GEO) database. Due to the small sample sizes in a single dataset and discrepancies of the characteristics within multiple heterogeneous datasets, it is necessary to integrate those massive datasets through systems biology tools and finally receive the stable and credible results [10]. So far, comprehensive integrated analysis of gene expression datasets in $C D$ is still missing. Therefore, we executed integrated analysis method by using robust rank aggregation (RRA) method to detect the consistently significant differentially expressed genes (DEGs) from various expression profiling datasets [11]. In addition, we performed weighted gene co-expression network analysis (WGCNA) to categorise those DEGs into a number of functional modules, and followed by functional and pathway enrichment analysis [12]. Connectivity Map (CMap) represents a useful bioinformatic technique for establishing the connections among genes, drugs and diseases [13, 14]. CMap has been applied to discover drug action mechanisms and disease pathogenesis, as well as to assist in the 
108

identification of novel potential therapeutics $[15,16]$. The DEGs of CD and control intestinal tissues were used to query the CMap database, which includes more than 7000 expression profiles corresponding to 1309 drugs. In the current study, we used CMap to explore therapeutic drugs that could potentially be effective against CD.

Here, we screened the differentially expressed genes from the intestinal tissue microarray data of $C D$ patients and healthy people by means of bioinformatics technology, and obtained the signal pathways related to the disease through enrichment analysis. Moreover, the potential therapeutic drugs for CD were explored via the CMap database. The study will help to understand the pathogenesis of $C D$ and lay a theoretical foundation for molecular diagnosis and targeted therapy. 
127 Methods

128 Data Collection and eligibility criteria

129 The Probe signal data from the key word "Crohn's disease biopsie"

130 were downloaded from Gene Expression Omnibus (GEO)

131 database(http://www.ncbi.nlm.nih.gov/geo/). The selection criteria for

132 this study were displayed as follows: (a) gene expression datasets which

133 included gene microarray chip technology; (b) studies comparing gene

134 expressions between active crohn's disease and normal controls tissue in

135 human samples; (c) the number of samples in each gene expression

136 profiling dataset was no less than 6; (d) Processed data or raw data can

137 be used for reanalysis should be provided in these databases. Studies

138 not meeting these selection criteria were not remained in the analysis.

\section{RRA analysis}

141 Data preprocessing steps and statistical analysis were performed using R software(https://www.r-project.org/) and Bioconductor packages [17].

Microarray datasets were normalized using RMA [18]and differential

expression analysis was implemented using the limma R package [19].

145 The statistical operations were performed using the Robust Rank Aggreg package of R statistical software [11]. Genes were ranked by statistical 


\section{Screening of small drug molecules}

151 To explore potential therapeutic drugs against $C D$, the DEGs were

152 compare to the expression signatures of 1309 drugs in the CMap 153 database (https://portals.broadinstitute.org/cmap/) [13, 14]. The 154 upregulated and downregulated DEGs generated through the RRA 155 method that had a $|\log F C|>0.5$ were imported into the CMap database 156 for analysis. Compounds with negative connectivity scores, which 157 indicated that the corresponding drug may reverse the expression of the value $<0.05$ were recorded(Table 2 ).

\section{WGCNA}

163 Gene co-expression network was implemented by the WGCNA package in R [12]. GSE16879 which including 37 CD patients and 12 controls was used to construct the WGCNA analysis. To include a sufficient amount of genes into WGCNA, genes with $p<0.05$ and |logarithmic fold changes| $(|\log F C|)>0.14$ were chosen from the ranked gene list. When the soft threshold was defined as 11 , the scale-free topology index was $>0.9$. In the present study, we chose a minimum module size of 150 for the genes dendrogram and the minimum height for merging modules at 
0.15. The modules were randomly color-labeled.

172

\section{Functional enrichment analysis}

174 Meaningful co-expression modules were screened out for functional

175 enrichment analysis, performing by metascape (http://metascape.org)

176 [20]. Top ten clusters with their representative enriched terms were

177 selected.

178

179 Statistical analysis

180 Statistical analyses were performed using $\mathrm{R}$ software version 3.5.0. P < 
192

193

194

195

196

197

198

199

200

201

202

203

204

\section{Results}

\section{CD microarray datasets}

According to the screening criteria, 9 datasets in $C D$ were finally included in this study: GSE10714 [21], GSE16879 [22], GSE36807 [23], GSE9686 [24], GSE10616 [25], GSE75214 [26], GSE6731 [27], GSE52746 [28], GSE68570 [29] (Table1). The description of the studies was provided in Table 1, such as GSE number, authors and number of samples. The number of $C D$ patients in these studies ranged from 4 to 59 , and the number of controls ranged from 3 to 22 . A total of $179 \mathrm{CD}$ patients and 94 healthy controls were finally included in this study.

\section{Table 1 Summary of those 9 genome-wide gene expression datasets}

\section{involving CD patients}

GSE number Samples Source types Platform authors

\begin{tabular}{|c|c|c|c|c|}
\hline 1 & GSE10714 & $\begin{array}{l}4 \mathrm{CD} \text { patients } \\
\text { and } 3 \text { controls }\end{array}$ & GPL570 & $\begin{array}{l}\text { Galamb O, Sipos F, } \\
\text { Solymosi N, Spisák } \\
\text { S et al. }\end{array}$ \\
\hline & & colonic biopsies & & Arijs I, De Hertogh \\
\hline 2 & GSE16879 & $\begin{array}{l}\text { patients and and ileal mucosal } \\
12 \text { controls biopsy }\end{array}$ & GPL570 & $\begin{array}{l}\text { G, Lemaire K, } \\
\text { Quintens R et al. }\end{array}$ \\
\hline 3 & GSE36807 & $\begin{array}{l}13 \mathrm{CD} \\
\text { patients and } 7 \text { colon biopsies } \\
\text { controls }\end{array}$ & GPL570 & $\begin{array}{l}\text { Montero-Meléndez } \\
\text { T, Llor X, } \\
\text { García-Planella E, } \\
\text { Perretti M et al. }\end{array}$ \\
\hline 4 & GSE9686 & $\begin{array}{l}11 \mathrm{CD} \\
\text { patients and } 8 \text { colon biopsies } \\
\text { controls }\end{array}$ & $\begin{array}{l}\text { GPL576 } \\
0\end{array}$ & $\begin{array}{l}\text { Carey R, Jurickova } \\
\text { I, Ballard E, } \\
\text { Bonkowski E et al. }\end{array}$ \\
\hline & GSE10616 & colon biopsies & GPL576 & Kugathasan S, \\
\hline
\end{tabular}




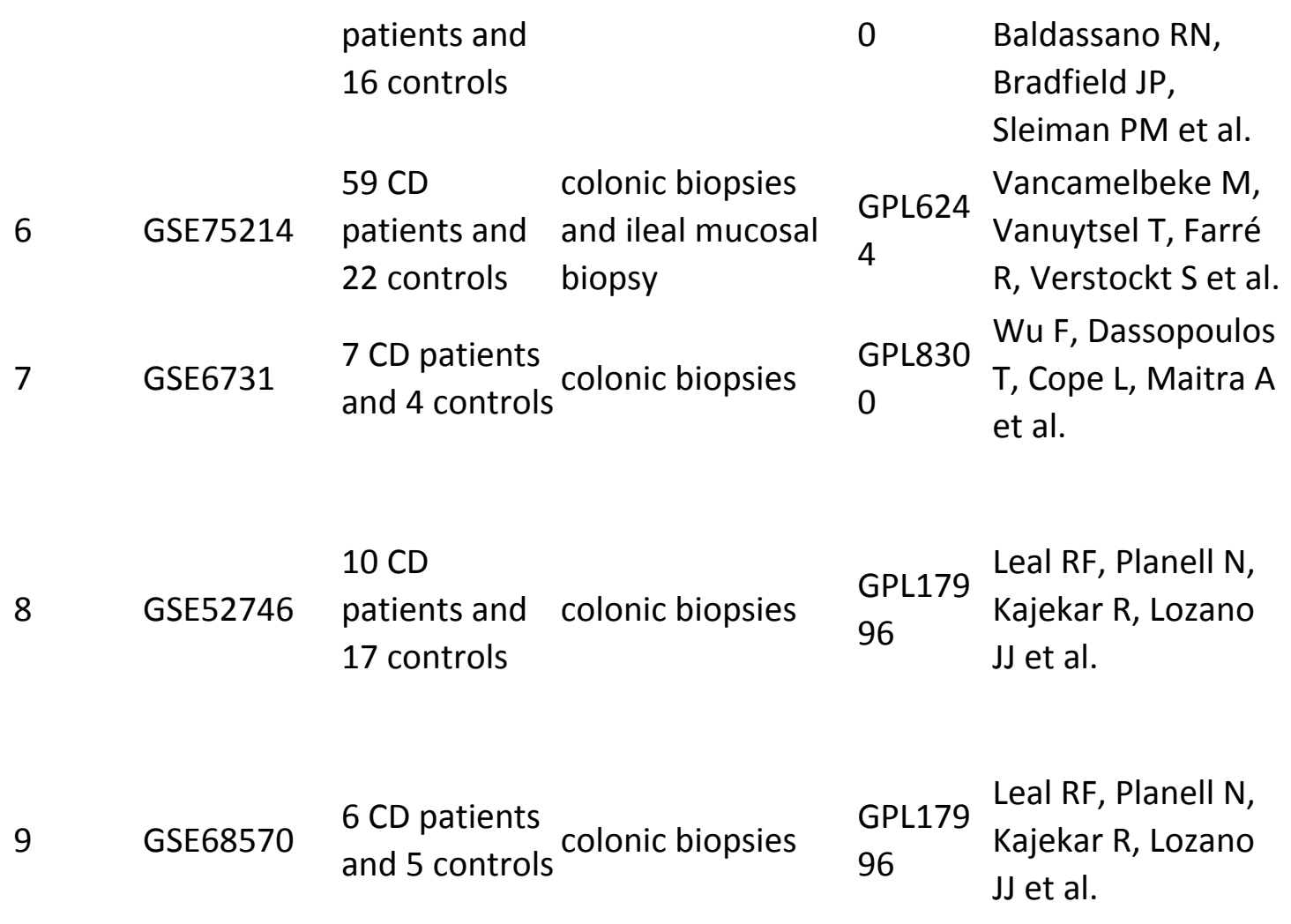

\section{RRA analysis}

By using a RRA method to integrate 9 datasets, upregulated and downregulated ranked gene lists were obtained. S1 Table showed those top 150 upregulated and top 23 downregulated genes in CD patients. These top 150 upregulated genes had a $\mathrm{p}<7.4 \mathrm{E}-8$ and a $|\operatorname{logFC}|>1$. These top 23 downregulated genes had a $\mathrm{p}<2.1 \mathrm{E}-10$ and a $|\operatorname{logFC}|>1$. The top 20 upregulated and downregulated genes in the integrated microarray analysis were showed in Fig 1 . Among these top genes, the abnormal expression of some genes had been well confirmed, such as MMP1, CLDN8 $[30,31]$. However, the roles of other genes in CD have not been previously reported in the literature, such as PCK1, CHP2 . 
column represents one dataset and each row represents one gene.

219 Numbers in each rectangle show the logarithmic fold change of genes in

220 each dataset. Green indicates decreased gene expression and red

221 indicates increased gene expression.

\section{Small drug molecule screening}

224 The related small molecules with highly significant correlations were

225 listed in Table 2. Among these molecules, tetracycline showed minimum

$226 \mathrm{p}$ value and higher negative correlation, indicating the potential

227 molecular signature that may counteract those of CD.

\section{Table 2 Results of CMap analysis}

\begin{tabular}{|c|c|c|c|}
\hline & & enrichr & \\
\hline cmap name & mean & $\mathrm{t}$ & $p$ \\
\hline tetracycline & -0.41 & -0.9 & 0.00004 \\
\hline $\begin{array}{l}\text { methoxamine } \\
\text { tubocurarine }\end{array}$ & -0.282 & -0.835 & 0.00135 \\
\hline chloride & -0.542 & -0.83 & 0.00151 \\
\hline $\begin{array}{l}\text { Prestwick-675 } \\
\text { benzbromaro }\end{array}$ & -0.265 & -0.818 & 0.00203 \\
\hline $\begin{array}{l}\text { ne } \\
\text { methocarbam }\end{array}$ & -0.301 & -0.874 & 0.00403 \\
\hline ol & -0.28 & -0.831 & 0.00959 \\
\hline colistin & -0.266 & -0.724 & 0.0118 \\
\hline disopyramide & -0.348 & -0.714 & 0.01361 \\
\hline
\end{tabular}




$\begin{array}{llll}\text { amantadine } & -0.349 & -0.682 & 0.02222 \\ \text { pepstatin } & -0.274 & -0.638 & 0.04144\end{array}$

\section{WGCNA}

232 The expressions of 5666 DEGs generated from the RRA analysis were 233 used for WGCNA, all of which had a $p<0.05$ and a $|\operatorname{logFC}|$ of $>0.14$. As 234 shown in Fig 2A, a power value of 11 was selected as the 235 soft-thresholding to ensure a scale-free network. We set 150 as the least 236 gene number of each gene network and 0.15 as the threshold for the merge of similar module. The WGCNA elucidated 7 coexpressed modules ranging in size from 269 to 1730 and the grey module represented a gene set that was not assigned to any of the modules (Fig 2B). Interactions of the 7 co-expression modules were analyzed (Fig 2C). The genes in each module were listed in S2 Table.

Fig 2 Plots in the WGCNA analysis using gene expressions in 37 CD of different soft-thresholding powers. The left panel displayed the

246 influence of soft-threshold power on scale-free topology fit index; the 247 right panel showed the influence of soft-threshold power on the mean connectivity. (B) Cluster dendrogram of coexpression genes and 
and was shown in different color. (C) Visualizing the gene network using a heatmap plot. Different colors of horizontal axis and vertical axis represented different modules.Light color represents low overlap and gradually dark red represents higher overlap.

Identifying the modules most correlated with clinical features has a great biological significance. As shown in Fig 3, black, blue, red and yellow modules positively correlated to $C D(p<0.05)$. Turquoise and brown modules negatively correlated to $C D(p<0.05)$. Brown and grey modules were positively correlated with a better clinical response to infliximab $(p<0.05)$ while blue and yellow modules was correlated negatively to treatment. Combined with Fig 4 ,we found that these six modules yielded two main clusters; one contained four modules (black, blue, red and yellow module) while the other contained two modules (brown and turquoise module). Moreover, two pairs of module combination had much higher adjacency degree and they are blue module and yellow module, black module and red module respectively.

The black module showed the highest correlation with $C D(r=0.71, p=$ 1e-8, Fig 3). The genes in black module mainly enriched in Interferon Signaling and defense response to virus. Genes in red module were enriched in Cell Cycle, cell division and cell cycle phase transition. Genes in blue module were enriched in several signaling pathways, including 
272 extracellular matrix organization, inflammatory response, blood vessel

273 development and leukocyte migration. The yellow module was similar to

274 the blue module, mainly enriched lymphocyte activation, adaptive

275 immune response, leukocyte activation involved in immune response.

276 Some other signaling pathways were observed to enrich in other

277 modules, such as monocarboxylic acid metabolic process, cofactor

278 metabolic process and Metabolism of lipids in turquoise, Transport of

279 small molecules in yellow. The main findings for the functional

280 enrichment analysis were shown in Table 3.

281

Table 3 Pathway and process enrichment analysis of those functional

283 coexpression modules in CD

\begin{tabular}{|c|c|c|c|c|c|c|}
\hline \multirow[t]{2}{*}{ Modules } & GO & Category & Description & Count & $\%$ & $\log 10(P)$ \\
\hline & R-HSA-91353 & Reactome & Interferon & 36 & 13.9534883721 & $-32.6634376812-28.3520653194$ \\
\hline \multirow{11}{*}{$\begin{array}{l}\text { Black } \\
\text { module }\end{array}$} & 1 & Gene Sets & Signaling & & & \\
\hline & GO:0051607 & GO Biological & Idefense response & 29 & 11.2403100775 & $-21.6512199059-18.4190287902$ \\
\hline & & Processes & to virus & & & \\
\hline & GO:0002476 & GO Biological & lantigen processing & & 2.71317829457 & $-12.8199138524-9.68463274966$ \\
\hline & & Processes & and presentation & & & \\
\hline & & & of endogenous & & & \\
\hline & & & peptide antigen via & & & \\
\hline & & & MHC class Ib & & & \\
\hline & R-HSA-98316 & Reactome & Class I MHC & 24 & 9.3023255814 & $-11.3182005651-8.38063594571$ \\
\hline & 9 & Gene Sets & $\begin{array}{l}\text { mediated antigen } \\
\text { processing \& } \\
\text { presentation }\end{array}$ & & & \\
\hline & GO:0001817 & $\begin{array}{l}\text { GO Biological } \\
\text { Processes }\end{array}$ & $\begin{array}{l}\text { Iregulation of } \\
\text { cytokine }\end{array}$ & 29 & 11.2403100775 & $-9.93175256791-7.09750146082$ \\
\hline
\end{tabular}


production

GO:0045088 GO Biological regulation of

$7.36434108527-7.5778005841-4.91964073607$

Processes innate immune

response

GO:0032496 GO Biological response to

$17 \quad 6.58914728682-7.10900753576-4.49660517829$

Processes lipopolysaccharide

M161

Canonical

PID IFNG

7

$2.71317829457-6.58416324878-4.03473422999$

Pathways

PATHWAY

GO:0060330 Processes

6

$2.32558139535-6.58267859823-4.03473422999$

response to interferon-gamma

GO:0036498 GO BiologicalIRE1-mediated 8

Processes unfolded protein

$3.1007751938-6.43708025362-3.91809958131$ response

Turquoise

GO:0032787 GO Biologicalmonocarboxylic

Processes acid metabolic

process

GO:0051186 GO Biological cofactor metabolic 115

Processes

R-HSA-55683 Reactome

process

3 Gene Sets lipids

GO:0044283 GO Biologicalsmall molecule 125

Processes biosynthetic

process

module

GO:0017144 GO Biologicaldrug metabolic

Processes process

GO:0006820 GO Biologicalanion transport 107

Processes

GO:0008202 GO Biologicalsteroid metabolic 70

Processes process

GO:0006091 GO Biologicalgeneration of

Processes precursor

metabolites and

energy

GO:0005975 GO Biologicalcarbohydrate 96

Processes metabolic process

R-HSA-21185 Reactome Biological

$9 \quad$ Gene Sets oxidations

GO:0030198 GO Biological extracellular

Processes matrix

Blue

module organization

GO:0006954 GO Biologicalinflammatory

Processes response
$1237.33890214797-28.1238108256-23.8124384638$

$6.861575179-25.2673411673-21.2569988012$

$1347.99522673031-23.0687643444-19.456361987$

$7.45823389021-19.2949845842-16.0356832879$

$7.75656324582-19.0211189435-15.8858378408$

$6.38424821002-18.265073659-15.2089738023$

$4.17661097852-16.9725530488-13.9833999817$

$87 \quad 5.19093078759-16.1087777292-13.1398280482$

$5.72792362768-14.3452501064-11.5109989993$

$51 \quad 3.04295942721-13.0028239955-10.2825162407$

$116 \quad 8.41189267585-55.1296356537-50.8182632918$

$149 \quad 10.8049311095-38.8989948741-35.1896825036$ 
GO:0001568 GO Biologicalblood vessel

Processes development

GO:0050900 GO Biologicalleukocyte

Processes migration

M5885 Canonical NABA MATRISOME131

Pathways ASSOCIATED

GO:0030155 GO Biological regulation of cell 116

Processes adhesion

GO:0001816 GO Biologicalcytokine

Processes production

GO:0002274 GO Biologicalmyeloid leukocyte 111

Processes activation

GO:0009611 GO Biological response to

114

Processes wounding

R-HSA-14742 Reactome Degradation of the 49

$28 \quad$ Gene Sets

143

106

120 extracellular
$7.68672951414-32.8831539596-29.4748715848$

$9.49963741842-28.6809763948-25.448785279$

$8.41189267585-26.555324351-23.4200432482$

$8.70195794054-25.7212283729-22.6139759938$

$8.049311095 \quad-24.9327449$

$-21.8518214596$

$8.26686004351-24.8377171008-21.7816172441$

$3.55329949239-24.5416313487-21.5090125878$

matrix

R-HSA-16401 Reactome Cell Cycle

97

$24.1895261845-62.4943601224-58.1829877606$

$70 \quad$ Gene Sets

GO:0051301 GO Biologicalcell division

$73 \quad 18.2044887781-40.4973015535-36.6630504464$

Processes

GO:0044770 GO Biologicalcell cycle phase 61

Processes transition

GO:0006260 GO BiologicalDNA replication 36

Processes

Red

module

R-HSA-69306 Reactome

DNA Replication 26

Gene Sets

GO:0006281 GO BiologicalDNA repair

R-HSA-69275 Reactome

Gene Sets

GO:0051321 GO Biologicalmeiotic cell cycle 29

GO:0071103 GO Biological DNA conformation 30

hsa04110 KEGG Cell cycle

GO:0046649 GO Biologicallymphocyte

Yellow GO:0002250 GO Biologicaladaptive immune 51

module Processes response

GO:0002366 GO Biologicalleukocyte
Processes

G2/M Transition 28

Processes Processes change

Pathway

$80 \quad 15.2671755725-32.6589516941-28.3475793323$

Processes activation

52

Processes activation involved

in immune

$45 \quad 11.2219451372-17.2330094157-14.489838778$

$6.9825436409-17.1069967286-14.3866889739$

$7.2319201995-15.6001764778-12.9877741204$

$7.4812967581-15.1235789271-12.5525692548$

$5.23690773067-14.4868416002-11.94632125$

$9.73282442748-15.1514204069-11.9539913974$

$9.92366412214-13.4023570595-10.4132039925$ 


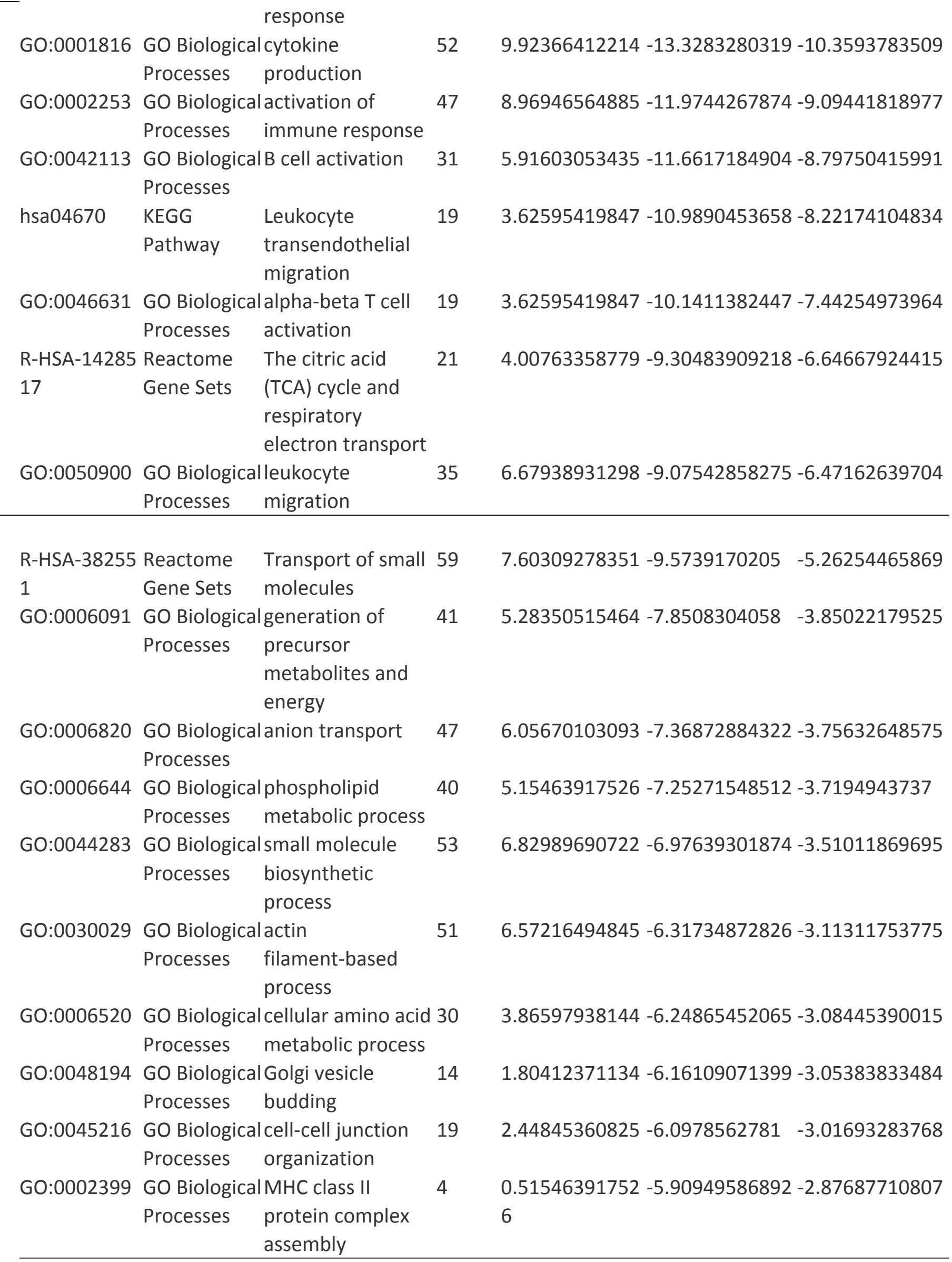




\section{Discussion}

285 Crohn's disease is a chronic inflammatory disorder which usually Lead to 286 pathological changes at different locations along the length 287 of gastrointestinal tract. The precise cause of $C D$ has not been determined. In this study, we integrated the gene expression data to shed light on the pathological mechanism of CD.

A large number of remarkably upregulated and downregulated genes were identified through RRA analysis, some of which have been reported in previous literature in CD, such as MMP1, MMP3, REG1B , REG1A, CLDN8, GUCA2A. Previous studies have reported that the with CD compared with control [32]. MMP1 and MMP3 belong to the family of metal dependent enzymes, which are capable of degrading a wide range of extracellular matrix components [33]. It is of note that MMP1 was involved in inflammation and have been implicated in tissue injury and repairing processes in CD [34]. In accordance with previous reports, the present study found that MMP-1 and MMP-3 were located in the blue module, which was mainly enriched in extracellular matrix organization and inflammatory response. Because MMP-1 and MMP-3 are the top two of upregulated differentially expressed genes ranking, over expression of MMP-1 and MMP-3 are probably key factors in the 
were highly expressed in CD colonic mucosa [35]. However, the molecular mechanism underlying how REG1B and REG1A exert their role in CD has not been determined yet. CLDN8 is a member of the family of claudins, which mainly located in the cell membrane and associated with tight junctions of cell adhesion [36]. It has been reported that CLDN8 might play a significant role in the injury of intestinal epithelial barrier of CD [37]. The expression level of CLDN8 was remarkably downregulated in $\mathrm{CD}$ colonic mucosa compared to normal controls, but the molecular mechanism underlying its role remains unclear. As previously demonstrated, GUCA2A expression was markedly decreased in $C D$, but its molecular mechanism is poorly understood [38]. The other genes (such as PCK1,CHP2) are novel gene signatures of $C D$, and there is still lack of researches on its role in $C D$ pathogenesis. Therefore, their aberrantly expressions need to be validated in future researches.

Several small molecules with potential therapeutic efficacy against CD were identified according to the CMap database. As a result of our screening effort, tetracycline has been identified as a potential therapeutic target for CD. Tetracycline were discovered in the 1940s and showed broad spectrum inhibitory activity against a wide range of microorganisms. Previous findings indicated that tetracycline exert pleiotropic functions independent of their antimicrobial activity, which include inhibition of MMPs, angiogenesis and inflammation [39]. Owing 
to the ability to inhibit MMPs expression and treat overgrowth of bacteria in the small intestine, tetracycline may be prescribed to treat CD. We might suppose that tetracycline could play certain roles to combat $C D$; however, further investigation on a large population is required to firmly establish the therapeutic effect in CD.

The WGCNA which is a kind of bioinformatics analysis method was conducted to explore the molecular mechanisms of various disease [40, 41]. Several reports involved IBD had been studied by this method [42, 43]. In this study, $5666 \mathrm{CD}$ associated genes generated from RRA analysis were used in WGCNA and most of them were classified into 7 co-expression modules. Six of the modules have significant associations with disease (Fig 3), which can be enriched more refined in three cluster (Fig 4), highlighting some new insights into the pathogenesis of CD. To further understand the importance of those modules in the pathogenesis of $C D$, enrichment analysis was performed using metascape. The two most critical modules, black module and blue module, which have the most significant relationship with disease need to be addressed. Black module was mainly enriched in some biological processes, the most important of which were Interferon Signaling, defense response to virus (Table 3). It is generally known that IFN-gamma plays a key role in the early steps of installation of inflammation, promoting monocyte recruitment and activation, and 
inducing the expression of other inflammatory cytokines. Interferon Signaling has been identified as a central aspect of innate immune response which induces a wide variety of antiviral proteins against pathogens infection $[44,45]$. Furthermore, previous studies have shown that Epstein-Barr virus and cytomegalovirus were the two main viruses that are positively correlated with the onset of CD [46]. In addition, bacteriophage was other viral agent that has been suspected to play a role in $C D$ pathogenesis [47]. Although there was not enough evidence that the virus is directly involved in $C D$, the study by Cadwell et al. [48]suggested that the combination of host genetic susceptibility and the presence of viral factors could lead to CD occurrence. What's more, several studies have found that for some CD patients with steroid resistance, antiviral therapy may be effective for relieving symptoms and improving the sensitivity of anti-inflammatory drugs [49]. From what have been discussed above, we may get the hypothesis that for some $C D$ patients, especially those with steroid-refractory $C D$, virus infection and the sequential Interferon Signaling pathway may be the key factor in the initial stage of disease onset. Similarly, the enrichment analysis of genes in the salmon module mainly involved in extracellular matrix organization, inflammatory response and blood vessel development. Meanwhile, yellow module clustered with blue module mainly enriched in the pathway of lymphocyte activation. The pathways enriched from 
372 the two important modules were all belong to the intermediate link of

373 the inflammatory response process, which explains the general

374 pathogenesis of $C D$, an autoimmune disease. Moreover, extracellular

375 matrix organization and blood vessel development also play a key role in

376 the process of intestinal fibrosis at the late stage of CD [50]. Intestinal

377 fibrosis continues to develop after intestinal primary injury and

378 inflammatory response are subsided [51]. As we know, severe intestinal

379 fibrosis is the main cause of intestinal resection, which seriously affects

380 the prognosis of patients. In other words, extracellular matrix

381 organization, inflammatory response and blood vessel development are

382 key factors in the development of CD.

Fig 3. Heatmap of the correlation between module eigengenes and

clinical traits of CD. The table is color coded by correlation according to the The table was color coded by correlation according to the color legend Each cell contains the corresponding correlation and p-value

Fig 4. Heatmap plot of the adjacencies of modules. The change of color

389 from blue (0) to red (1) in the heatmap represented the connectivity 390 degree of different modules from weak to strong. 


\section{Conclusion}

In conclusion, this study identified a number of key genes and co-expression biologically functional modules which may be involved in the progress of $C D$, based on differentially expressed genes between $C D$ samples and normal mucosas. Additionally, the present study screened a small drug molecule named tetracycline, which might be exploited as adjuvant drug for improved therapeutics for CD. Our findings suggest that virus infectious may be the initial link in CD. Moreover, extracellular matrix organization, inflammatory response and blood vessel development are the key pathways for the development of $C D$, which not only participate in the stage of inflammatory response process, but also play a key role in the late phase of intestinal fibrosis. This point may provide a new idea for improving the prognosis of $C D$.

(1)




\section{Acknowledgments}

416 This work did not receive any specific funding from any sources.

417

418

419

420

421

422

423

424

425

426

427

428

429

430

431

432

433

434

435

436 


\section{Author Contributions}

438 Conceptualization: Zheng Wang, Jie Zhu, Lixian Ma

439 Data curation: Zheng Wang, Jie Zhu

440 Formal analysis: Zheng Wang, Jie Zhu, Lixian Ma

441 Investigation: Zheng Wang, Jie Zhu, Lixian Ma

442 Methodology: Zheng Wang

443 Software: Zheng Wang

444 Supervision: Lixian Ma

445 Visualization: Zheng Wang

446 Writing-original draft: Zheng Wang, Jie Zhu

447 Writing-review \& editing: Zheng Wang, Jie Zhu, Lixian Ma 


\section{References}

459 1. Torres J, Mehandru S, Colombel JF, Peyrin-Biroulet L. Crohn's disease. Lancet (London, England).

460 2017;389(10080):1741-55. http://dx.doi.org/10.1016/s0140-6736(16)31711-1. PMID: 27914655

461 2. Molodecky NA, Soon IS, Rabi DM, Ghali WA, Ferris M, Chernoff G, et al. Increasing incidence and

462 prevalence of the inflammatory bowel diseases with time, based on systematic review.

463 Gastroenterology. 2012;142(1):46-54.e42; quiz e30. http://dx.doi.org/10.1053/j.gastro.2011.10.001.

464 PMID: 22001864

465 3. Moller FT, Andersen V, Wohlfahrt J, Jess T. Familial risk of inflammatory bowel disease: a

466 population-based cohort study 1977-2011. The American journal of gastroenterology.

468 4. Baumgart DC, Carding SR. Inflammatory bowel disease: cause and immunobiology. Lancet

469 (London, England). 2007;369(9573):1627-40. http://dx.doi.org/10.1016/s0140-6736(07)60750-8.

470 PMID: 17499605

471 5. Zhang YZ, Li YY. Inflammatory bowel disease: pathogenesis. World journal of gastroenterology.

472 2014;20(1):91-9. http://dx.doi.org/10.3748/wjg.v20.i1.91. PMID: 24415861

473 6. Hugot JP, Chamaillard M, Zouali H, Lesage S, Cezard JP, Belaiche J, et al. Association of NOD2

474 leucine-rich repeat variants with susceptibility to Crohn's disease. Nature. 2001;411(6837):599-603.

475 http://dx.doi.org/10.1038/35079107. PMID: 11385576

476 7. Fritz T, Niederreiter L, Adolph T, Blumberg RS, Kaser A. Crohn's disease: NOD2, autophagy and ER

477 stress converge. Gut. 2011;60(11):1580-8. http://dx.doi.org/10.1136/gut.2009.206466. PMID: 
479 8. Massey DC, Parkes M. Genome-wide association scanning highlights two autophagy genes,

480 ATG16L1 and IRGM, as being significantly associated with Crohn's disease. Autophagy.

481 2007;3(6):649-51. PMID: 17921695

482 9. Liu JZ, van Sommeren S, Huang H, Ng SC, Alberts R, Takahashi A, et al. Association analyses

483 identify 38 susceptibility loci for inflammatory bowel disease and highlight shared genetic risk across

484 populations. Nature genetics. 2015;47(9):979-86. http://dx.doi.org/10.1038/ng.3359. PMID:

\section{$485 \quad \underline{26192919}$}

10. Rung J, Brazma A. Reuse of public genome-wide gene expression data. Nature reviews Genetics.

11. Kolde R, Laur S, Adler P, Vilo J. Robust rank aggregation for gene list integration and

(Oxford,

England).

2012;28(4):573-80.

490

491

492

http://dx.doi.org/10.1093/bioinformatics/btr709. PMID: 22247279

12. Langfelder $\mathrm{P}$, Horvath S. WGCNA: an R package for weighted correlation network analysis. BMC bioinformatics. 2008;9:559. http://dx.doi.org/10.1186/1471-2105-9-559. PMID: $\underline{19114008}$

13. Lamb J, Crawford ED, Peck D, Modell JW, Blat IC, Wrobel MJ, et al. The Connectivity Map: using gene-expression signatures to connect small molecules, genes, and disease. Science (New York, NY). 2006;313(5795):1929-35. http://dx.doi.org/10.1126/science.1132939. PMID: 17008526

14. Lamb J. The Connectivity Map: a new tool for biomedical research. Nature reviews Cancer. 2007;7(1):54-60. http://dx.doi.org/10.1038/nrc2044. PMID: 17186018

15. Gheeya J, Johansson P, Chen QR, Dexheimer T, Metaferia B, Song YK, et al. Expression profiling identifies epoxy anthraquinone derivative as a DNA topoisomerase inhibitor. Cancer letters. 
501

502

503

504

505

506

507

508

509

510

16. Song Y, Liu J, Huang S, Zhang L. Analysis of differentially expressed genes in placental tissues of preeclampsia patients using microarray combined with the Connectivity Map database. Placenta. 2013;34(12):1190-5. http://dx.doi.org/10.1016/j.placenta.2013.09.013. PMID: 24125805

17. Gentleman RC, Carey VJ, Bates DM, Bolstad B, Dettling M, Dudoit S, et al. Bioconductor: open software development for computational biology and bioinformatics. Genome biology. 2004;5(10):R80. http://dx.doi.org/10.1186/gb-2004-5-10-r80. PMID: 15461798

18. Bolstad BM, Irizarry RA, Astrand M, Speed TP. A comparison of normalization methods for high density oligonucleotide array data based on variance and bias. Bioinformatics (Oxford, England). 2003;19(2):185-93. PMID: 12538238

19. Smyth GK. Linear models and empirical bayes methods for assessing differential expression in microarray experiments. Statistical applications in genetics and molecular biology. 2004;3:Article3. http://dx.doi.org/10.2202/1544-6115.1027. PMID: 16646809

20. Tripathi S, Pohl MO, Zhou Y, Rodriguez-Frandsen A, Wang G, Stein DA, et al. Meta- and Orthogonal Integration of Influenza "OMICs" Data Defines a Role for UBR4 in Virus Budding. Cell host \& microbe. 2015;18(6):723-35. http://dx.doi.org/10.1016/j.chom.2015.11.002. PMID: 26651948

21. Galamb O, Sipos F, Solymosi N, Spisak S, Krenacs T, Toth K, et al. Diagnostic mRNA expression patterns of inflamed, benign, and malignant colorectal biopsy specimen and their correlation with peripheral blood results. Cancer epidemiology, biomarkers \& prevention : a publication of the American Association for Cancer Research, cosponsored by the American Society of Preventive Oncology. 2008;17(10):2835-45. http://dx.doi.org/10.1158/1055-9965.epi-08-0231. PMID: 18843029

22. Arijs I, De Hertogh G, Lemaire K, Quintens R, Van Lommel L, Van Steen K, et al. Mucosal gene expression of antimicrobial peptides in inflammatory bowel disease before and after first infliximab 
523

524

treatment. PloS one. 2009;4(11):e7984. http://dx.doi.org/10.1371/journal.pone.0007984. PMID:

\section{$\underline{19956723}$}

23. Montero-Melendez T, Llor X, Garcia-Planella E, Perretti M, Suarez A. Identification of novel predictor classifiers for inflammatory bowel disease by gene expression profiling. Plos one. 2013;8(10):e76235. http://dx.doi.org/10.1371/journal.pone.0076235. PMID: 24155895

24. Carey R, Jurickova I, Ballard E, Bonkowski E, Han X, Xu H, et al. Activation of an IL-6:STAT3-dependent transcriptome in pediatric-onset inflammatory bowel disease. Inflammatory bowel diseases. 2008;14(4):446-57. http://dx.doi.org/10.1002/ibd.20342. PMID: 18069684

25. Kugathasan S, Baldassano RN, Bradfield JP, Sleiman PM, Imielinski M, Guthery SL, et al. Loci on $20 q 13$ and $21 q 22$ are associated with pediatric-onset inflammatory bowel disease. Nature genetics. 2008;40(10):1211-5. http://dx.doi.org/10.1038/ng.203. PMID: 18758464

26. Vancamelbeke M, Vanuytsel T, Farre R, Verstockt S, Ferrante M, Van Assche G, et al. Genetic and Transcriptomic Bases of Intestinal Epithelial Barrier Dysfunction in Inflammatory Bowel Disease. Inflammatory bowel diseases. 2017;23(10):1718-29.

\section{http://dx.doi.org/10.1097/mib.0000000000001246. PMID: 28885228}

27. Wu F, Dassopoulos T, Cope L, Maitra A, Brant SR, Harris ML, et al. Genome-wide gene expression differences in Crohn's disease and ulcerative colitis from endoscopic pinch biopsies: insights into distinctive pathogenesis. Inflammatory bowel diseases. 2007;13(7):807-21. http://dx.doi.org/10.1002/ibd.20110. PMID: 17262812

28. Leal RF, Planell N, Kajekar R, Lozano JJ, Ordas I, Dotti I, et al. Identification of inflammatory mediators in patients with Crohn's disease unresponsive to anti-TNFalpha therapy. Gut. 
30. von Lampe B, Barthel B, Coupland SE, Riecken EO, Rosewicz S. Differential expression of matrix metalloproteinases and their tissue inhibitors in colon mucosa of patients with inflammatory bowel active Crohn's disease. Gut. 2007;56(1):61-72. http://dx.doi.org/10.1136/gut.2006.094375. PMID: evolution, gene regulation and functional analysis in mouse models. Biochimica et biophysica acta. 
and

biophysics

reports.

2017;12:198-205.

http://dx.doi.org/10.1016/j.bbrep.2017.10.003. PMID: 29090282 cross-talk between the IL23 pathway and the intestinal barrier in inflammatory bowel disease. gastroenterology. 2015;50(10):1241-52. http://dx.doi.org/10.3109/00365521.2015.1038849. PMID:

39. Amin AR, Attur MG, Thakker GD, Patel PD, Vyas PR, Patel RN, et al. A novel mechanism of action of tetracyclines: effects on nitric oxide synthases. Proceedings of the National Academy of Sciences of the United States of America. 1996;93(24):14014-9. PMID: 8943052

581 diagnostic biomarkers of metastatic melanoma. Plos one. 2018;13(1):e0190447. 
586

587

42. Xie D, Zhang Y, Qu H. Crucial genes of inflammatory bowel diseases explored by gene expression profiling analysis. Scandinavian journal of gastroenterology. 2018;53(6):685-91. http://dx.doi.org/10.1080/00365521.2018.1461923. PMID: 29909694

43. Lin X, Li J, Zhao Q, Feng JR, Gao Q, Nie JY. WGCNA Reveals Key Roles of IL8 and MMP-9 in Progression of Involvement Area in Colon of Patients with Ulcerative Colitis. Current medical science. 2018;38(2):252-8. http://dx.doi.org/10.1007/s11596-018-1873-6. PMID: 30074183

44. Noisakran S, Carr DJ. Type I interferons and herpes simplex virus infection: a naked DNA approach as a therapeutic option? Immunologic research. 2001;24(1):1-11. http://dx.doi.org/10.1385/ir:24:1:01. PMID: 11485206

45. Su C, Zhan G, Zheng C. Evasion of host antiviral innate immunity by HSV-1, an update. Virology journal. 2016;13:38. http://dx.doi.org/10.1186/s12985-016-0495-5. PMID: 26952111

46. Wagner J, Sim WH, Lee KJ, Kirkwood CD. Current knowledge and systematic review of viruses associated with Crohn's disease. Reviews in medical virology. 2013;23(3):145-71. http://dx.doi.org/10.1002/rmv.1720. PMID: 22674582

47. Riley PA. Bacteriophages in autoimmune disease and other inflammatory conditions. Medical hypotheses. 2004;62(4):493-8. http://dx.doi.org/10.1016/j.mehy.2003.12.016. PMID: 15050095

48. Cadwell K, Patel KK, Maloney NS, Liu TC, Ng AC, Storer CE, et al. Virus-plus-susceptibility gene interaction determines Crohn's disease gene Atg16L1 phenotypes in intestine. Cell. 2010;141(7):1135-45. http://dx.doi.org/10.1016/j.cell.2010.05.009. PMID: 20602997

49. Ormeci AC, Akyuz F, Baran B, Soyer OM, Gokturk S, Onel M, et al. Steroid-refractory inflammatory bowel disease is a risk factor for CMV infection. European review for medical and pharmacological sciences. 2016;20(5):858-65. PMID: 27010142 
bioRxiv preprint doi: https://doi.org/10.1101/543835; this version posted February 7, 2019. The copyright holder for this preprint (which was not certified by peer review) is the author/funder, who has granted bioRxiv a license to display the preprint in perpetuity. It is made available under aCC-BY 4.0 International license.

608

609

610

611

612

613

614

615

616

617

618

619

620

621

622

623

624

625

626

627

628

629

50. Speca S, Giusti I, Rieder F, Latella G. Cellular and molecular mechanisms of intestinal fibrosis.

World journal of gastroenterology. 2012;18(28):3635-61. http://dx.doi.org/10.3748/wjg.v18.i28.3635.

PMID: 22851857

51. Valatas V, Filidou E, Drygiannakis I, Kolios G. Stromal and immune cells in gut fibrosis: the myofibroblast and the scarface. Annals of gastroenterology. 2017;30(4):393-404.

http://dx.doi.org/10.20524/aog.2017.0146. PMID: 28655975

(1)

(1)

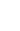

(1)

(1)

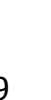




\section{Supporting information}

631 S1 Table. Top 150 up-regulated and top 23 down-regulated genes in CD

632 patients.

633 S2 Table. Six main functional co-expression modules and related genes

634 involved in CD.

635

636

637

638

639 

Scale independence

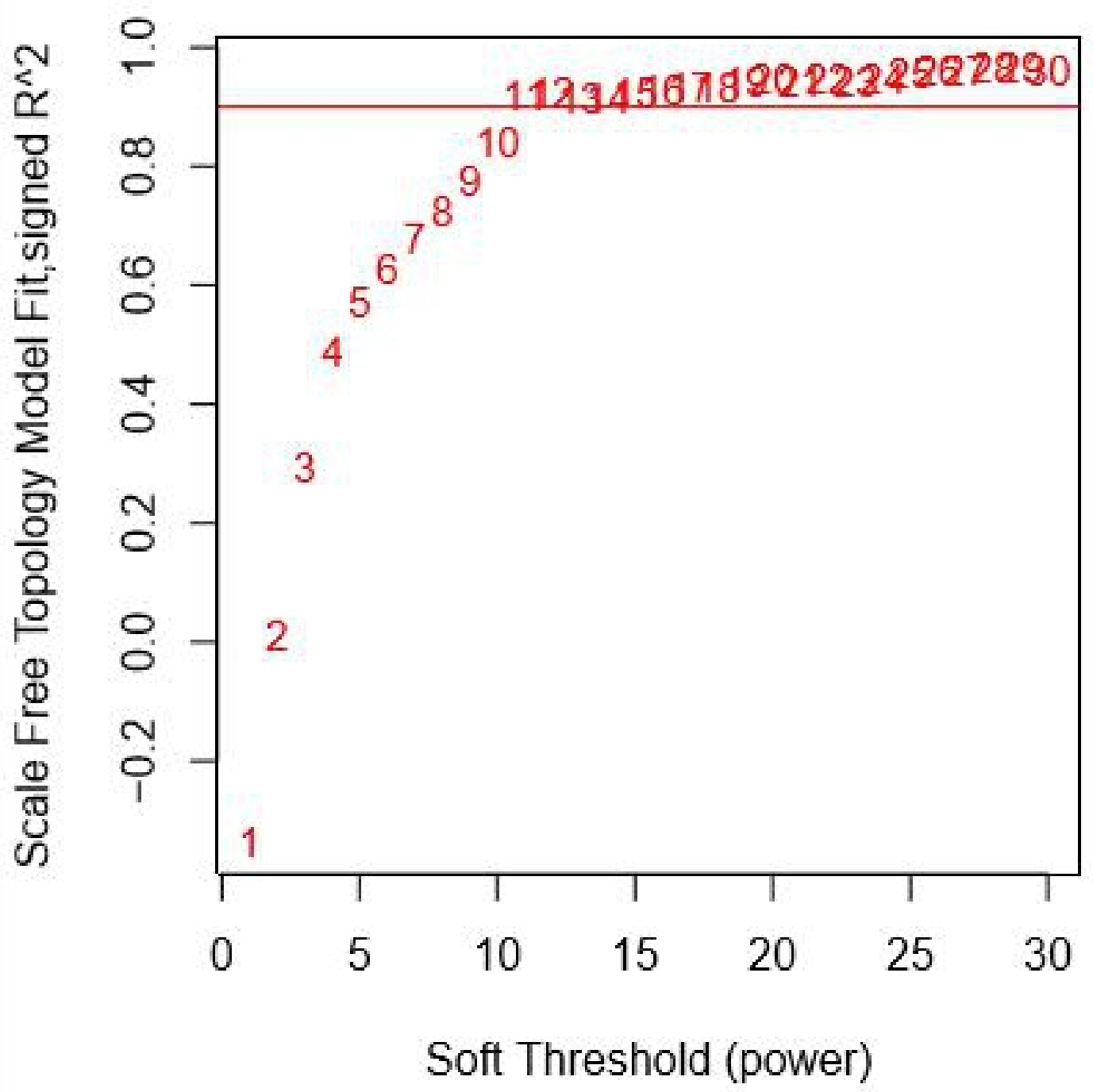

Mean connectivity

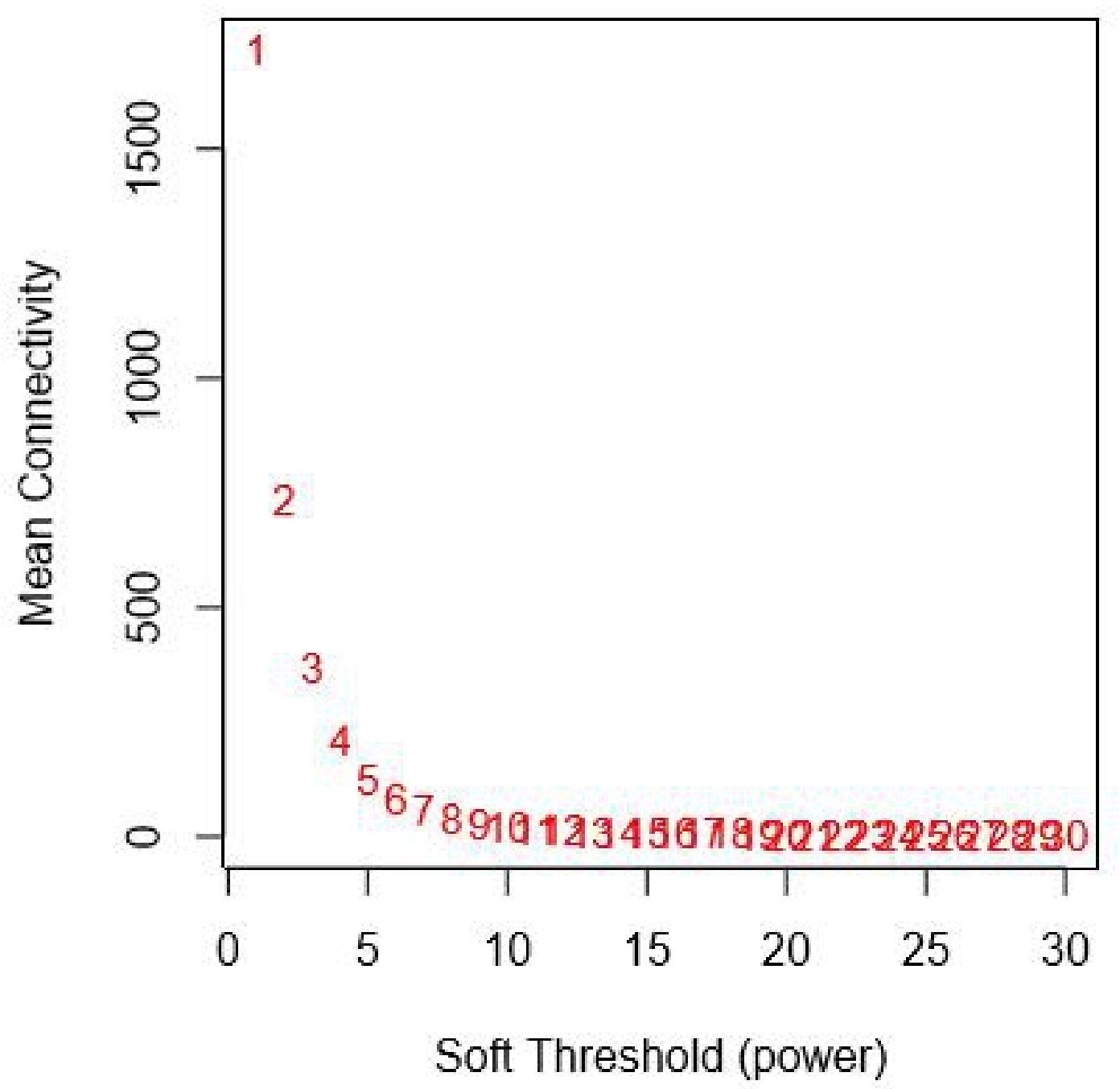

Fig2A 
Network heatmap plot, all genes

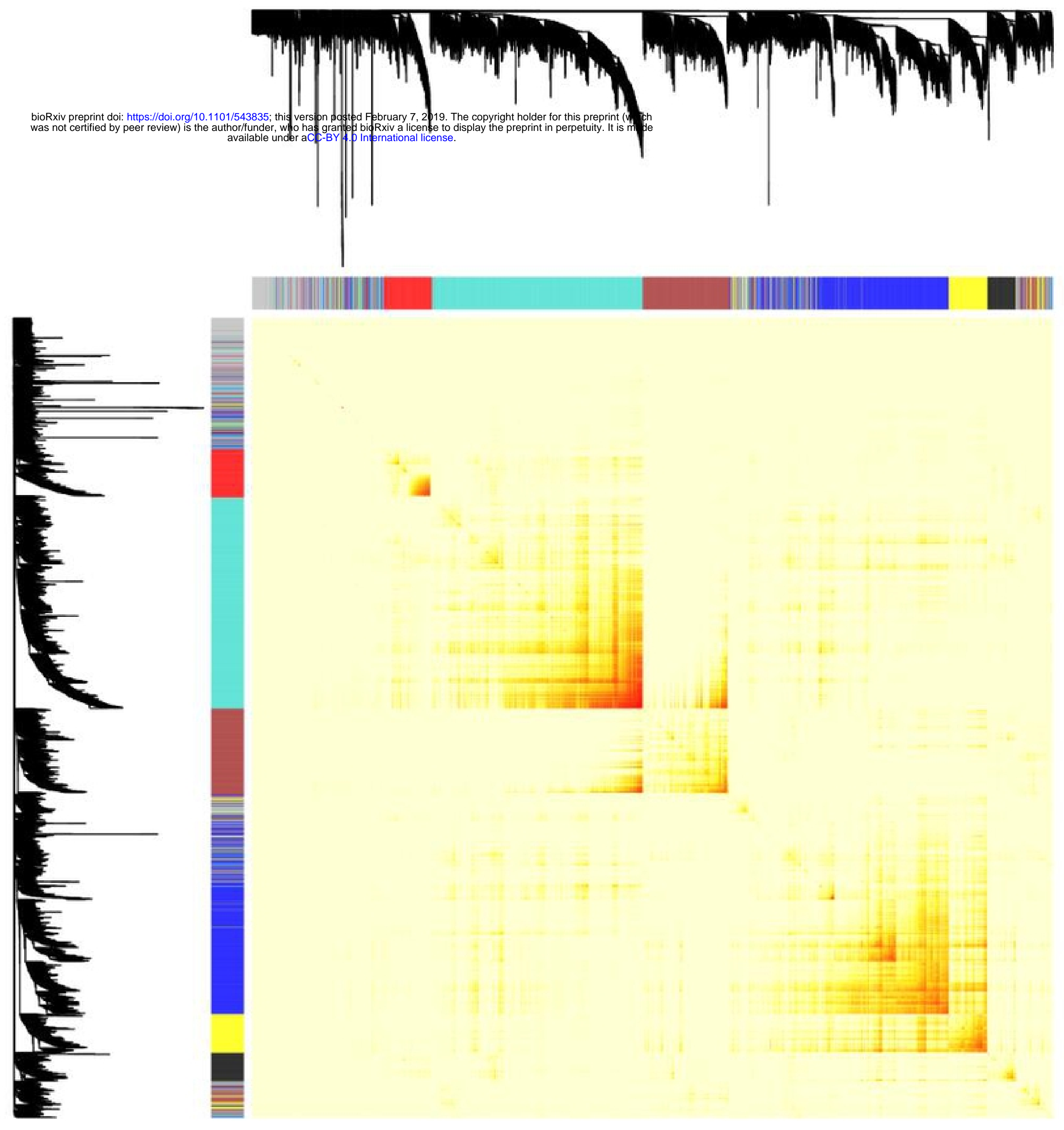

Fig2C 


\section{Module-trait relationships}

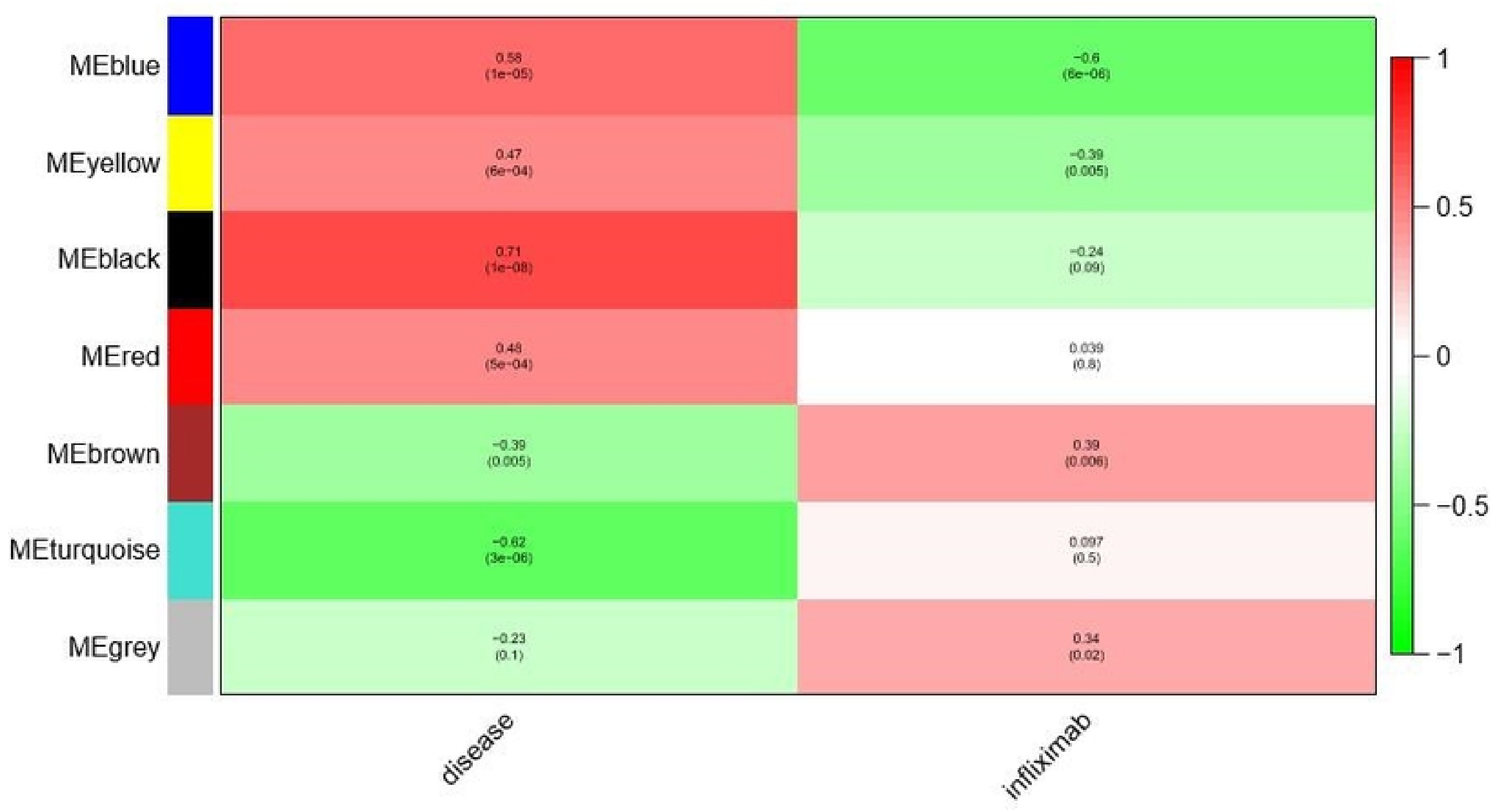

Fig3 

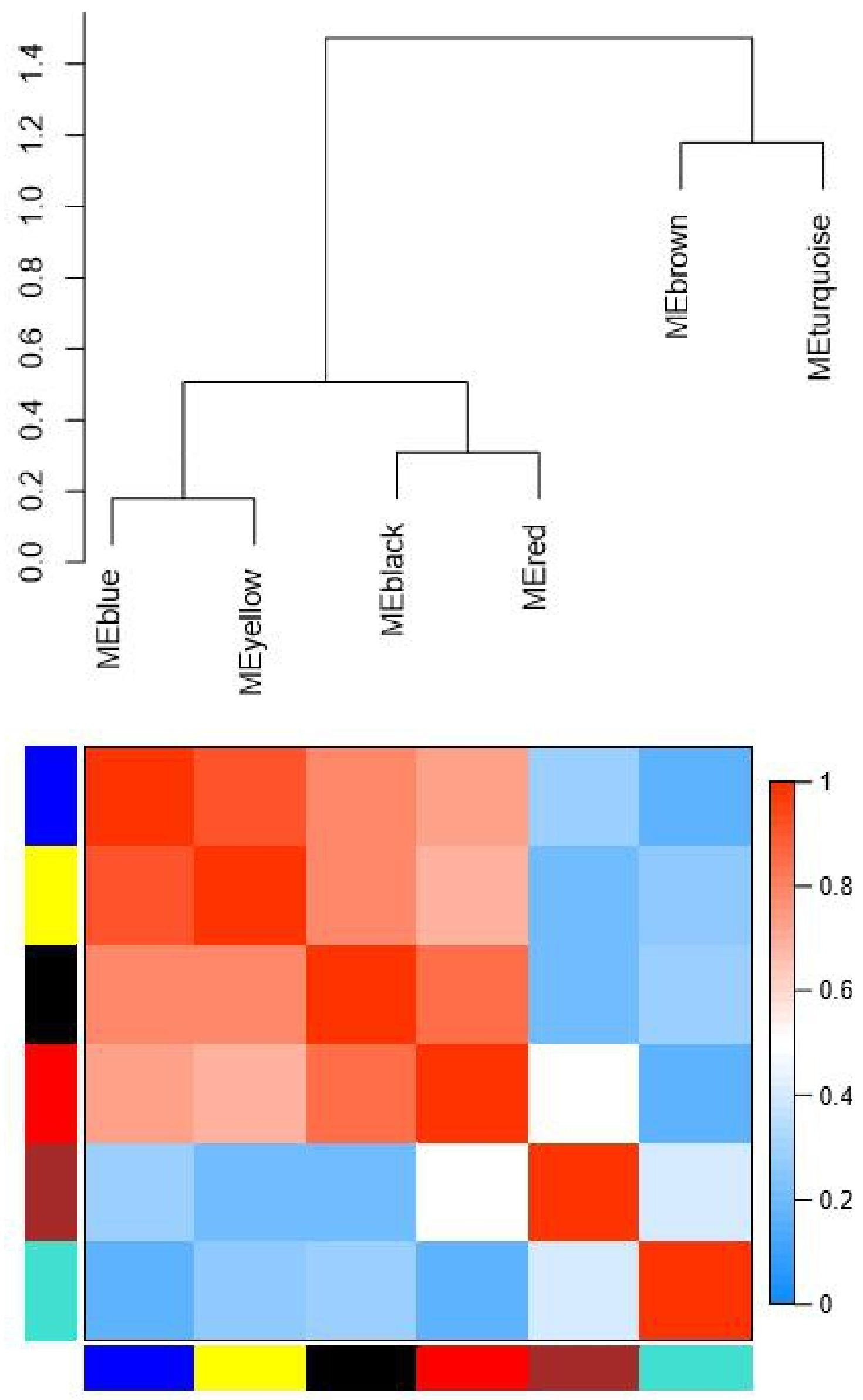

Fig4 